\title{
Cationic Copolymerization of 1,3-Dioxolane and Isoprene
}

\author{
Masahiko Okada, Shigeru IkaI, Kazumasa Chiba, Masahiro Hrrota, and Yuya Yamashita \\ The Department of Synthetic Chemistry, Faculty of Engineering, Nagoya University, Chikusa-ku, Nagoya, Japan.
} (Received July 16, 1969)

\begin{abstract}
Copolymerization of 1,3-dioxolane(DOL) and isoprene(Ip) by $\mathrm{BF}_{3} \cdot \mathrm{Et}_{2} \mathrm{O}$ has been studied, particularly laying stress on the microstructure of the products. The diad fractions determined by NMR analysis have indicated that both monomer units are distributed randomly in the copolymer chain. The loss of unsaturation (the fraction of saturated Ip units to total Ip units), which was generally observed in cationically prepared Ip homopolymers, diminished with increasing DOL fraction and became practically negligible in the copolymers rich in DOL units. The structure of Ip units in the copolymer was also found to depend on the composition, namely, the fraction of the 1,4-structure, which was prevailing in Ip homopolymers prepared by the cationic initiator, decreased markedly with increasing DOL fraction in the copolymer.
\end{abstract}

KEY WORDS Cationic Copolymerization / 1,3-Dioxolane / Isoprene / Boron Trifluoride / Microstructure / Nuclear Magnetic Resonance /

Recently copolymerization of cyclic monomers with vinyl monomers has attracted much attention from the viewpoints of elucidating the copolymerization mechanism of these monomers of intrinsically different reactivities on one side, and of improving the properties of the polymers obtained by ring opening polymerization of cyclic monomers on the other. A number of copolymerization experiments with various pairs of cyclic and vinyl monomers has disclosed that a random copolymer consisting of cyclic and vinyl monomer units can be formed only from cyclic monomers, the propagating species of which in cationic polymerization have, at least partly, a carbonium ionic character. For instance, 1,3dioxolane, the active species of which is most reasonably assumed to be a resonance hybrid of carbonium ion and tertiary oxonium ion, copolymerizes with styrene to yield a copolymer in which both monomer units are almost randomly distributed. $^{1}$ On the other hand, 3,3-bis(chloromethyl)oxacyclobutane which propagates through oxonium ions does not cause random copolymerization with vinyl monomers such as isobutyl vinyl ether, styrene and $\alpha$-methyl styrene. ${ }^{2,16}$ In addition to the carbonium ionic nature of the active chain ends, another important factor for the formation of a random copolymer is, of course, that crossover propagations from cyclic monomers to vinyl monomers and vice-versa must occur at a comparable rate as homopropagations. For example, the copolymerization of isobutyl vinyl ether and 1,3-dioxepane resulted in a copolymer in which both monomer units formed blocks of considerably long sequences, ${ }^{3}$ owing to the infrequent occurrence of the crossover propagations.

There have been a number of papers and patents which dealt with copolymerization of cyclic and vinyl monomers, but few of them have described in detail the structure of the products. Fractionation by suitable solvents and elemental analysis data on the fractionated materials alone do not provide confirmative proof for the formation of a random copolymer. A definitive conclusion in this regard could be drawn from the determination of the sequence distribution of monomer units in a polymer chain which, apparently, is composed of cyclic and vinyl monomers. In fact, as described above, we have previously studied the sequence distribution of monomer units in the copolymer of 1,3-dioxolane(DOL) and styrene(St) using NMR and hydrolytic techniques and confirmed the formation of an almost random copolymer. ${ }^{1}$ The successful preparation of such a random copolymer of DOL and $\mathrm{St}$ prompted us to carry out similar experiments on the copolymerization of DOL with dienes which have moderate reactivity in cationic polymerization. The present paper describes the determina- 
tion of the sequence distribution of monomer units in the copolymer of DOL and isoprene(Ip) by means of NMR and the effects of DOL on the structure of Ip units in the copolymer.

\section{RESULTS AND DISCUSSION}

A typical N.M.R. spectrum of DOL-Ip copolymer is shown in Figure 1. The complicated peaks appearing on both sides of the sharp singlet at $\tau 6.35$ which is assigned to the oxyethylene protons (a) of DOL recurring units are characteristic of the copolymer. The peaks at $\tau$ 5.9-6.3 may reasonably be associated with the methylene (c) or methine $\left(c^{\prime}\right)$ protons of Ip units which are formed by the attachment of DOL monomers to an active chain end of Ip, since these protons should appear in a lower magnetic field than the oxyethylene protons (a) of DOL recurring units because of a deshielding effect of the adjacent carbon-carbon double bond.

$$
\begin{gathered}
-\left(-\mathrm{OCH}_{2} \mathrm{CH}_{2} \mathrm{OCH}_{2}\right)_{\bar{n}} \\
\text { a } \quad \text { a } \quad \text { b }
\end{gathered}
$$

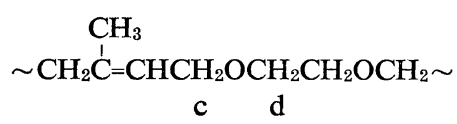

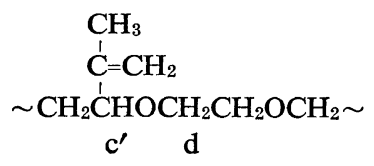

Therefore the appearance of these peaks can be taken as an evidence for the occurrence of crosspropagation from Ip to DOL. The protons (d) in the above structures are expected to appear in a slightly higher magnetic field than the oxyethylene protons of DOL recurring units and partly account for the multiplet peaks appearing $\tau 6.4$ 6.6. The rest of the peaks in this region can be assigned to the methylene protons (e) and (f) of DO Lunits which are formed by the cross-propagation from DOL to Ip.

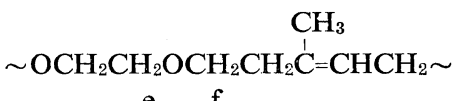

$$
\begin{aligned}
& \begin{array}{c}
\multicolumn{4}{c}{\mathrm{CH}_{3}} \\
\sim \mathrm{OCH}_{2} \mathrm{CH}_{2} \mathrm{OCH}_{2} \mathrm{CH}_{2} \stackrel{\mathrm{C}}{\sim} \sim \\
\text { e f f } \quad \stackrel{\mathrm{C}}{\mathrm{CH}}=\mathrm{CH}_{2}
\end{array}
\end{aligned}
$$

Thus, it is confirmed by the NMR spectrum that both cross-propagations occur during the copolymerization of DOL and Ip, in other words, a real copolymer is formed.

Furthermore, the determination of oxymethylene protons (b) which appear at $\tau 5.3$ provides useful information on the distribution of both monomer units in the copolymer chain. It is clear from the structural formulae (4) and (5) that, when the active chain end of DOL attacks Ip monomers to form a covalent bond, the oxymethylene protons of the DOL unit are converted to the protons (f) which no longer appear at $\tau 5.3$. That is, the oxymethylene protons of the DOL monomer unit remain as such only when the active chain end of

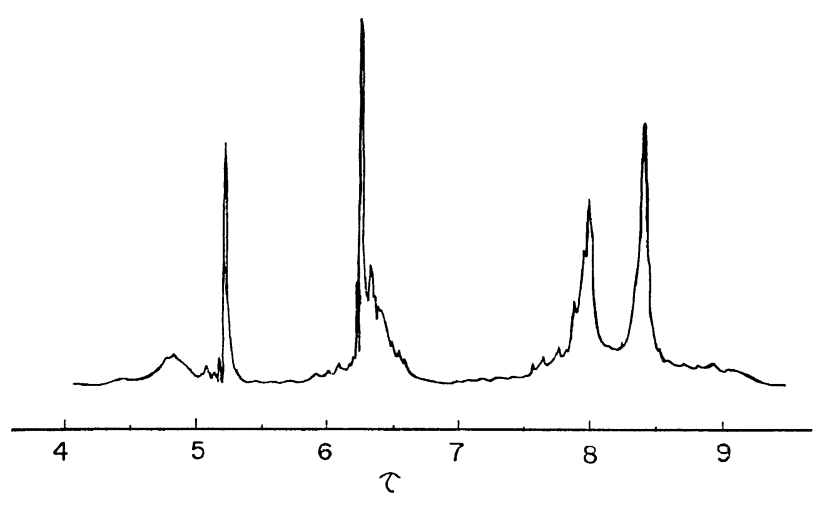

Figure 1. NMR spectrum of DOL-Ip copolymer.

Solvent: Chloroform, Temp. $60^{\circ} \mathrm{C}$, Conc. $10 \%$, Copolymer composition: DOL $43.0 \mathrm{~mol} \%$, Ip $57.0 \mathrm{~mol} \%$. 
DOL adds DOL monomers, and hence, these protons correspond to the diad of two consecutive DOL units. One can easily calculate the diad fraction $\{D D\}$ from the relative peak area of the oxymethylene protons and the copolymer composition can be determined by elemental analysis. In an ordinary copolymer consisting of $\mathrm{A}$ and $\mathrm{B}$ monomer units, as long as it is not of a very low molecular weight, the diad fraction $\{\mathrm{AB}\}$ generally equals the diad fraction $\{\mathrm{BA}\}$. Accordingly in the present case, the diad fractions $\{\mathrm{DI}\},\{\mathrm{ID}\}$ and $\{\mathrm{II}\}$ can be estimated using the following equations.

$$
\begin{aligned}
& \{\mathbf{D}\}=\{\mathbf{D D}\}+\{\mathbf{D I}\} \\
& \{\mathbf{I}\}=\{\mathbf{I D}\}+\{\mathbf{I I}\}
\end{aligned}
$$

where $\{\mathbf{D}\}$ and $\{\mathbf{I}\}$ denote the molar fraction of DOL and Ip units in the copolymer, respectively. Average sequence lengths of both monomer units are given as follows:-

$$
\bar{l}_{D}=\frac{\{\mathrm{D}\}}{\{\mathrm{DI}\}} \quad \text { and } \quad \bar{l}_{I}=\frac{\{\mathrm{I}\}}{\{\mathrm{ID}\}}
$$

Also, run number $R^{4}$ which refers to the number of sequences per 100 monomer units can be calculated.

$$
R=200\{\mathrm{DI}\}
$$

These values characterize the distribution of monomer units in the copolymer and the results on the DOL-Ip copolymers prepared with varying monomer ratios in feed are summarized in Tables I and II.

If the monomer reactivity ratios of this copolymerization are known, one can calculate theoretically diad fractions, the run number of the copolymer and average sequence lengths of both mono- mer units assuming that both monomer units are statistically incorporated into a copolymer chain according to the monomer reactivity ratios. The copolymer composition curve obtained at low conversion (within $10 \%$ ) is shown in Figure 2. However, appropriate monomer reactivity ratios which fitted well the experimental curve over the whole range could not be found. This abnormality can be explained by taking the equilibrium polymerization of DOL into account: The polymerization of DOL involves an equilibrium between monomer and polymer ${ }^{5-9}$. Hence the apparent reactivity of DOL should be reduced with decreasing DOL fraction in feed since the rate of DOL monomer consumption is proportional to $\left([\mathrm{M}]-[\mathrm{M}]_{e}\right)$, although the absolute value of equilibrium mono-

Table I. Copolymerization of DOL and Ip

\begin{tabular}{lccccc}
\hline & $\begin{array}{c}\text { DOL } \\
\text { molar } \\
\text { Run } \\
\text { frac- } \\
\text { No. } \\
\text { tion in } \\
\text { feed }\end{array}$ & $\begin{array}{c}\text { Conver- Carbon } \\
\text { sion } \\
(\%)\end{array}$ & $\begin{array}{c}\text { content } \\
(\%)\end{array}$ & $\begin{array}{c}\text { DOL } \\
\text { molar } \\
\text { frac- } \\
\text { tion in } \\
\text { copoly- } \\
\text { mer }\end{array}$ & MW \\
\hline 31 & 0.90 & 77.6 & 50.5 & 0.95 & $3.3 \times 10^{3}$ \\
32 & 0.80 & 55.2 & 51.5 & 0.92 & $2.7 \times 10^{3}$ \\
33 & 0.70 & 43.9 & 53.2 & 0.88 & $3.4 \times 10^{3}$ \\
34 & 0.60 & 30.7 & 57.2 & 0.77 & $3.0 \times 10^{3}$ \\
35 & 0.50 & 20.4 & 61.2 & 0.66 & $3.2 \times 10^{3}$ \\
36 & 0.40 & 18.4 & 67.2 & 0.51 & $3.3 \times 10^{3}$ \\
37 & 0.30 & 19.0 & 70.5 & 0.43 & $2.9 \times 10^{3}$ \\
38 & 0.20 & 19.0 & 75.2 & 0.31 & $2.4 \times 10^{3}$ \\
39 & 0.10 & 36.6 & 81.7 & 0.15 & $2.0 \times 10^{3}$ \\
$40^{b}$ & 0.00 & 34.3 & 88.2 & - & $3.3 \times 10^{3}$ \\
\hline
\end{tabular}

a Total monomers $0.05 \mathrm{~mol}$, Initiator $\mathrm{BF}_{3} \cdot \mathrm{Et}_{2} \mathrm{O}$ $0.001 \mathrm{~mol}$, Solvent $\mathrm{CH}_{2} \mathrm{Cl}_{2} 1.0 \mathrm{ml}$, Temperature $0^{\circ} \mathrm{C}$, Time 5 days

${ }^{\text {b }}$ polymerized at $-78^{\circ} \mathrm{C}$ to avoid gel-formation.

c calculated: $88.7 \%$.

Table II. Diads distribution, average chain lengths and run number $R$ of DOL-Ip copolymers

\begin{tabular}{ccccccccc}
\hline $\begin{array}{c}\text { Run } \\
\text { No. }\end{array}$ & $\{\mathrm{D}\}$ & $\{\mathrm{I}\}$ & $\{\mathrm{DD}\}$ & $\begin{array}{c}\{\mathrm{DI}\} \\
=\{\mathrm{ID}\}\end{array}$ & $\{\mathrm{II}\}$ & $\overline{l_{D O L}}$ & $\bar{l}_{I p}$ & $R$ \\
\hline 31 & 0.95 & 0.05 & 0.91 & 0.04 & 0.01 & 23 & 1.3 & 8 \\
32 & 0.92 & 0.08 & 0.85 & 0.07 & 0.01 & 13 & 1.1 & 14 \\
33 & 0.88 & 0.12 & 0.78 & 0.10 & 0.02 & 8.8 & 1.2 & 20 \\
34 & 0.77 & 0.23 & 0.60 & 0.17 & 0.06 & 4.5 & 1.4 & 34 \\
35 & 0.66 & 0.34 & 0.41 & 0.25 & 0.09 & 2.6 & 1.4 & 50 \\
36 & 0.51 & 0.49 & 0.31 & 0.20 & 0.29 & 2.5 & 2.5 & 40 \\
37 & 0.43 & 0.57 & 0.26 & 0.17 & 0.40 & 2.5 & 3.4 & 34 \\
38 & 0.31 & 0.69 & 0.18 & 0.13 & 0.56 & 2.4 & 5.3 & 26 \\
39 & 0.15 & 0.85 & 0.06 & 0.09 & 0.76 & 1.7 & 9.4 & 18 \\
\hline
\end{tabular}




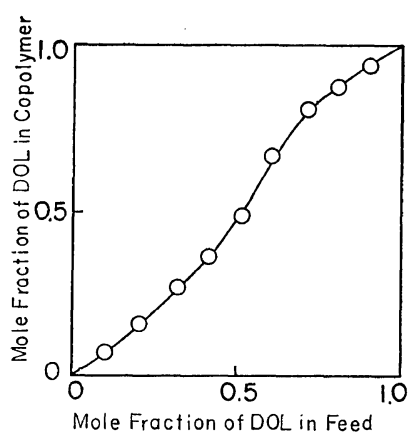

Figure 2. Copolymer composition curve of DOL-Ip copolymer.

Total monomers $0.05 \mathrm{~mol}, \quad \mathrm{BF}_{3} \cdot \mathrm{Et}_{2} \mathrm{O}$

$0.001 \mathrm{~mol}$, Solvent $\mathrm{CH}_{2} \mathrm{Cl}_{2} 2.0 \mathrm{ml}$, Temp. $0^{\circ} \mathrm{C}$.

mer concentration $[\mathrm{M}]_{e}$ in the copolymerization may differ from that in the homopolymerization and depends upon the molar fraction in feed. Though no comparison with theoretical values can be made, it may safely be assumed from the results in Table II that DOL and Ip monomer units are randomly distributed in the copolymer chain according to their reactivities.

It has been reported that cationic polymerization of isoprene yields, particularly at high conversion, insoluble cross-linked polymers concurrently with the low molecular weight soluble polymer. ${ }^{10}$ The conformation of the double bond of the soluble polymer is predominantly trans 1,4 and there is always some loss of unsaturation. ${ }^{11}$ In Figure 3, the loss of unsaturation which refers to the percent of the saturated Ip units to total Ip units in the copolymer is plotted against the

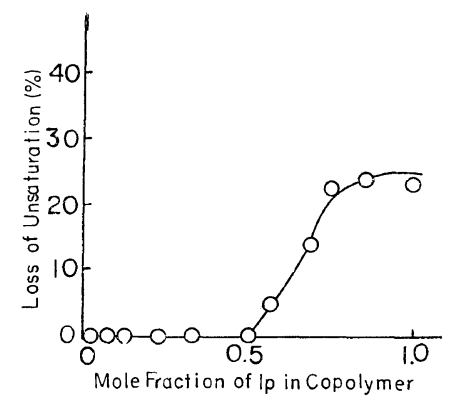

Figure 3. Variation of loss of unsaturation ${ }^{\mathrm{a}}$ with copolymer composition.

a See the text. molar fraction of Ip units in the copolymer. The homopolymer of Ip, examined for comparison, was found to contain $23 \%$ saturated units which was in agreement with the reported value of the polyisoprene prepared under a similar reaction condition. ${ }^{11}$ The loss of unsaturation of the copolymers, when the DOL fraction was below 0.25 , was nearly the same as that of polyisoprene. However, it diminished abruptly by further increasing the DOL fraction and there was practically no loss of unsaturation in the copolymers in which the DOL molar fraction was higher than 0.5. The formation of saturated Ip units may be accounted for by intramolecular transfer reactions accompanying cyclization, ${ }^{12}$ although there may be some other modes of reactions.

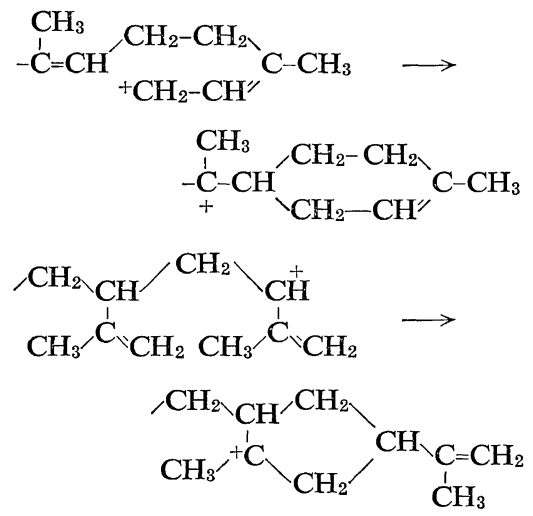

In fact, the NMR spectrum of the copolymers, having a high Ip content, showed methyl proton signals at $\tau 9.1$ which could be attributed to the methyl group on the saturated carbon atom in the above structures. For the occurrence of these cyclization reactions, at least the penultimate unit of the active chain end of Ip must be an Ip unit. The probability for this is of course reduced with increasing molar fraction of DOL in feed (See Table II). Moreover, it seems likely that the DOL monomer solvates the active chain end of the Ip unit to prevent intramolecular transfer reactions. As a consequence, every Ip unit in copolymers which are rich in DOL units, has, practically, one unsaturated linkage.

Figure 4 illustrates the variation of the fraction of the internal double bond to total double bonds with copolymer composition. The fraction of the internal double bond, which corresponds to trans 1,4-structure, decreases gradually with 


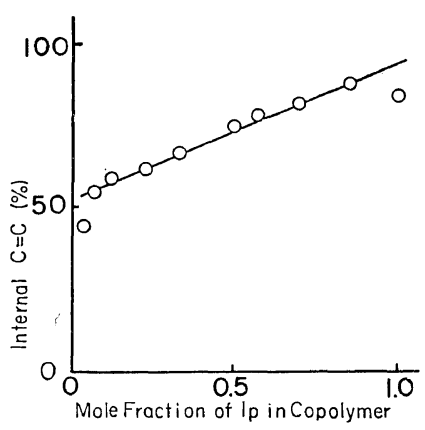

Figure 4. Variation of the fraction of internal double bond to total double bonds with copolymer composition.

decreasing molar fraction of Ip and approaches to 0.5. An attempt to estimate the fractions of the 1,2- and the 3,4-structures separately by infrared spectroscopy according to the procedure applied to polyisoprene ${ }^{13}$ was unsuccessful because of the low solubility of the copolymers, especially those of high DOL content, in carbon disulfide and of the overlapping of the absorbance due to DOL units. Qualitatively, the fraction of the 1,2-structure was 1.5 to 3 times greater than that of the 3,4-structure. The marked decrease in the fraction of the 1,4-structure with increasing molar fraction of $\mathrm{DOL}$ is difficult to explain. This is related to the question why a trans 1,4structure is prevailing in the Ip homopolymer prepared by the cationic initiator. Although there is no direct evidence at present, it could be interpreted on the assumption that the positive charge of the active chain end of the Ip unit largely localizes on the carbon atom at the end, while in the presence of DOL, it delocalizes by solvation, thus leading to the increase in the relative fractions of the 1,2- and 3,4-structures.

Previously we have succeeded in preparing the copolymers of DOL and styrene with molecular weights of $3 \times 10^{5} .^{14}$ In the present study, it was intended to synthesize high molecular weight copolymer of DOL and Ip utilizing the living nature of the polymerization of DOL. ${ }^{15}$ The results on the copolymerization carried out within a high vacuum system using $\mathrm{Et}_{3} \mathrm{OBF}_{4}$ as an initiator are given in Table III. Although the rate of copolymerization was extremely slow, a copolymer with molecular weight as high as $1.6 \times 10^{5}$ was successfully prepared when the molar ratio of DOL to Ip in feed was high. The molecular weight of the product does not differ much from the values calculated on the assumption that one polymer chain grows from one initiator molecule. This would indicate that chain transfer reaction hardly occurs during the copolymerization of DOL and Ip in the presence of a large excess of the former monomer.

\section{EXPERIMENTAL}

\section{Materials}

1,3-Dioxolane (DOL) was prepared and purified as described in the previous paper. ${ }^{15}$

Commercial isoprene(Ip) of Phillips Petroleum Co. was dried over calcium hydride and fractionally distilled.

Commercial methylene chloride and boron fluoride ethyl ether complex $\left(\mathrm{BF}_{3} \cdot \mathrm{Et}_{2} \mathrm{O}\right)$ were purified by the usual methods.

Triethyl oxonium tetrafluoroborate $\left(\mathrm{Et}_{3} \mathrm{OBF}_{4}\right)$ was prepared according to Meerwein's procedure. $^{17}$

\section{Copolymerization Procedure}

DOL, $\mathrm{Ip}$ and $\mathrm{BF}_{3} \cdot \mathrm{Et}_{2} \mathrm{O}$ dissolved in methylene chloride were charged in this order into an ampoule under a nitrogen atmosphere and after having been sealed, the ampoule was placed in an ice-bath. The copolymerization was terminated by adding a solution of methanolic ammonia containing a small amount of $\beta$-phenyl naphthylamine. The resulting solution was poured into a large excess of methanol kept at dry-ice temperature to precipitate copolymer as completely as

Table III. Copolymerization of DOL and Ip by $\mathrm{Et}_{3} \mathrm{OBF}_{4}{ }^{\mathrm{a}}$

\begin{tabular}{|c|c|c|c|c|c|c|c|}
\hline \multirow{2}{*}{ Run No. } & \multirow{2}{*}{$\begin{array}{l}\text { DOL } \\
(\mathrm{mol})\end{array}$} & \multirow{2}{*}{$\underset{(\mathrm{mol})}{\mathrm{Ip}}$} & \multirow{2}{*}{$\begin{array}{l}\text { Time } \\
\text { (day) }\end{array}$} & \multirow{2}{*}{$\begin{array}{l}\text { Yield } \\
(\%)\end{array}$} & \multicolumn{2}{|c|}{$\bar{M}_{n} \times 10^{-4}$} & \multirow{2}{*}{$\begin{array}{l}\text { Ip in } \\
\text { copolymer } \\
(\mathrm{mol} \%)\end{array}$} \\
\hline & & & & & obsd. & calcd. & \\
\hline DI-1 & 0.113 & 0.0205 & 14 & 43.0 & 16.1 & 11.4 & 5.3 \\
\hline DI-2 & 0.0988 & 0.0311 & 30 & 48.2 & 10.8 & 12.5 & 10.2 \\
\hline
\end{tabular}

a Solvent: none, Polymerization temp., Room temp., Initiator $\mathrm{Et}_{3} \mathrm{OBF}_{4} 3.71 \times 10^{-2} \mathrm{mmol}$ 
possible. The precipitate was separated by a centrifuge, dissolved in benzene and reprecipitated in methanol. The product was dried under reduced pressure at room temperature to constant weight. There may be a small loss of oligomers by this procedure, but it does not seem to affect the experimental results and the conclusion derived therefrom.

\section{Characterization}

Copolymer composition was determined by carbon analysis which was run on a Yanagimoto Micro Carbon and Hydrogen Analyzer.

Total unsaturation in the copolymer was determined by the iodine monochloride method $^{18}$ and the fraction of internal double bond was measured using perbenzoic acid according to the procedure described by Kolthoff et al. ${ }^{19}$

NMR spectra were recorded on a Japan Electron Optics Spectrometer C-60 working at $60 \mathrm{Mc}$ in chloroform at $60^{\circ} \mathrm{C}$. The oxymehtylene protons in the copolymer were determined from the relative peak area of the singlet signal appearing at 5.3. The error of the measurement is estimated to be within $5 \%$, depending upon the copolymer composition.

Number average molecular weight of the copolymer was determined by a Hitachi Vapor Pressure Osmometer or by a Mechrolab Membrane Osmometer 502 on solutions in benzene.

\section{REFERENCES}

1. M. Okada, Y. Yamashita, and Y. Ishii, Makromol. Chem., 94, 181 (1966).

2. S. Aoki, Y. Haruta, T. Otsu, and M. Imoto, Bull. Chem. Soc. Japan, 38, 1922 and 1928 (1965).
3. M. Okada and Y. Yamashita, Makromol. Chem, 126, 266 (1969).

4. H. J. Harwood and W. M. Ritchey, J. Polymer Sci., Part B 2, 601 (1964).

5. M. Kučera and J. Pichler, Vysokomol. Soedin. 7, 3 and 10 (1965).

6. P. H. Plesch and P. H. Westermann, Internat. Symp. on Macromolecules, Prague 1965, Preprint p. 506.

7. S. Gorin and L. Monnerie, Bull. Soc. Chim. France, 2047 (1966).

8. A. A. Brikenstein, A. F. Abrosimov, N. I. Astrakhantseva, and V.P. Volkov, Plastisckie Massy, 5, 7 (1967).

9. Y. Yamashita, M. Okada, K. Suyama, and H. Kasahara, Makromol. Chem., 114, 146 (1968).

10. C. A. Thomas and W. H. Carmody, J. Amer. Chem. Soc., 55, 3854 (1933).

11. W. S. Richardson, J. Polym. Sci., 13, 325 (1954).

12. W. Cooper in "The Chemistry of Cationic Polymerization" P. H. Plesch Ed., Pergamon Press, 1963, p. 357.

13. W. S. Richardson and A. Sacher, J. Polym. Sci., 10, 353 (1953).

14. Y. Yamashita, M. Okada, and M. Hirota, Makromol. Chem., 122, 284 (1969).

15. Y. Yamashita, M. Okada, and H. Kasahara, Makromol. Chem., 117, 256 (1968).

16. T. Higashimura, T. Masuda, and S. Okamura, J. Polym. Sci., Part A-1, 7, 1154 (1969).

17. H. Meerwein, E. Battenberg, H. Gold, E. Pfeil, and G. Willfang, J. Prakt. Chem., 154, 83 (1939).

18. T. S. Lee, I. M. Kolthoff, and M. Anne Mairs, J. Polym. Sci., 3, 66 (1948).

19. I. M. Kolthoff, T. S. Lee, and M. Anne Mairs, J. Polym. Sci., 2, 199 (1947). 